\title{
Using Single-Item Measures to Examine the Relationships between Work, Personality, and Well-Being in the Workplace
}

\author{
Gary M. Williams, Andrew P. Smith \\ Centre for Occupational and Health Psychology, School of Psychology, Cardiff University, Cardiff, UK \\ Email:smithap@cardiff.ac.uk
}

Received 3 March 2016; accepted 29 May 2016; published 1 June 2016

Copyright (C) 2016 by authors and Scientific Research Publishing Inc.

This work is licensed under the Creative Commons Attribution International License (CC BY). http://creativecommons.org/licenses/by/4.0/

c) (i) Open Access

\begin{abstract}
Measuring the well-being of employees through questionnaire measures can give a useful indication of the positive or negative mental health of a workforce along with their satisfaction with their circumstances. Furthermore, measuring the antecedents of these outcomes provides a basis for reducing negative outcomes and promoting positive mental health and satisfaction within an organization. This endeavour can quickly become impractical, however, as taking into account the range of possible environmental or personal factors, can lead to a lengthy and burdensome measurement tool. The current paper examines the use of single-items for this purpose, demonstrating that single-item measures of work-related and personality factors exhibit relationships with each other and with outcomes that the literature on well-being predicts. Using multiple-regression analysis, the results show that work related factors such as control and reward provide significant predictors of well-being outcomes including job satisfaction, while personality factors such as selfesteem and self-efficacy are significant predictors of all outcome measures. Furthermore, variations in the relationships with specific outcomes and interaction effects are found. The results suggest that using single-item measures may provide a valid approach to investigating well-being in the workplace in circumstances that may require very brief scales.
\end{abstract}

\section{Keywords}

Well-Being, Well-Being Process Questionnaire, Work Characteristics, Personality, Job Satisfaction, Affect, Anxiety and Depression 


\section{Introduction}

Accounting for employees' well-being is not only relevant to the mental and physical health of society in general, but is also occupationally relevant in terms of staff performance and attendance (SCMH, 2007). Although well-being has traditionally been measured as the absence of negative mental health outcomes such as depression or anxiety (Cheng \& Furnham, 2003), measurement of subjective well-being (SWB) also allows one to take into account an individual's perception of happiness or satisfaction with their circumstances (Diener, 1984).

Although SWB research is generally concerned with general positive mood and satisfaction with life as a whole, there are important reciprocal relationships between workplace well-being and SWB outcomes. For example, poor SWB itself has been linked to physical health outcomes such as cardiovascular disease and mortality, and mental health outcomes such as clinical depression (Gargiulo \& Stokes, 2009), which in turn can lead to absenteeism, presenteeism (i.e. reduced performance), and staff turnover (SCMH, 2007). Meanwhile workplace factors such as a high effort and high demands along with low reward or low control lead to poor SWB, particularly in terms of job satisfaction (Tsutsumi \& Kawakami, 2004; Van Der Doef \& Maes, 1999). Therefore, being able to assess the potential causes of poor well-being or antecedents of positive well-being in the workplace is of practical importance. Due to the tendency for studies to focus on only one element of well-being, and for the purpose of clarity, from here on the term "well-being" refers to the absence of negative outcomes and presence of positive SWB interchangeably, while "SWB" is used to specify instances where research has focused solely on happiness or subjective well-being (positive affect, negative affect, and satisfaction).

\subsection{Predicting Well-Being-Work}

When measuring well-being and its contributory factors in practice, the evidence from research suggests that numerous factors including environmental factors and personality factors play an important role (Diener, Suh, Lucas, \& Smith, 1999). In terms of the work environment, two of the most influential models in this domain are the Job-Demands-Control (-Support) (JDCS) model (Johnson \& Hall, 1988) and the Effort Reward Imbalance (ERI) model (Siegrist, 1996), both of which have been repeatedly tested and have support for their main hypotheses (Tsutsumi \& Kawakami, 2004; Van Der Doef \& Maes, 1999). In brief, the JDCS model suggests that the combination of high job demands (e.g. a large workload or limited time allowance) and low job control (e.g. ability to decide how to do the work) lead to negative outcomes (Karasek Jr., 1979), while adding low social support or isolation to the above relationship further increases the risk of negative effects (Johnson \& Hall, 1988). Although the model can be applied in different ways (for example with control or support as buffers), the job-strain (demands-control) and iso-strain (demands-control-support) hypotheses described above are more consistently supported (Van Der Doef \& Maes, 1999).

The ERI model is similar and complementary to the JDCS model, however the relationship of interest is that between effort (similar to demands described above) and reward (e.g. respect, financial reward, or opportunity for advancement (Rydstedt, Devereux, \& Sverke, 2007)). The ERI model can be used to suggest that the combination of high effort and low reward leads to negative health outcomes, although more in-depth applications of the model can be used to distinguish between types of effort (e.g. extrinsic vs. intrinsic) or reward (e.g. esteem, financial, promotion prospects) or to consider the increased susceptibility of those who score highly on an overcommitment factor (Siegrist et al., 2004). The JDCS model and ERI model have both been associated with numerous outcomes such as general health, cardiovascular disease, life satisfaction, depression and anxiety (Häusser, Mojzisch, Niesel, \& Schulz-Hardt, 2010; Tsutsumi \& Kawakami, 2004; Van Der Doef \& Maes, 1999). Based on the models' predictions, an organisation could expect to find that those employees who scored high on demands and low on control and support, or those who scored high on effort and low on reward, would be most at risk for negative outcomes (Karasek Jr., 1979; Siegrist, 1996).

\subsection{Predicting Well-Being-Personality}

The influence of personality has gained particular attention in the research on positive SWB. Early views of what made up a happy person included self-esteem, extroversion, and optimism (Wilson 1967), and several reviews since then have suggested that personality may be the major determinant of SWB (DeNeve \& Cooper, 1998). Top-down theories of SWB such as the dynamic equilibrium model (Headey \& Wearing, 1989) suggest 
that personality is a critical factor, potentially as a tendency to experience situations in a positive or negative way (Diener, 1984), or to return SWB to an equilibrium after life events have disturbed the balance (DeNeve \& Cooper, 1998). These top-down approaches to SWB suggest that personality may influence the relationships between circumstances and outcomes, as well as having direct effects themselves (Brief, Butcher, George, \& Link, 1993). In the case of organisational well-being, interaction theories could be interpreted to suggest that as well as having a direct effect, personality factors may influence the extent to which demands, effort, control, or reward have their effects on well-being. Those investigating the effects of personality on well-being have commonly focused on the "Big 5" personality factors, particularly neuroticism, however research has demonstrated the importance of personality beyond these factors. Self-efficacy, for example, has been linked to anxiety and depression symptoms (Bandura, 1997), as the perception of personal inefficacy to achieve desired outcomes, or deal with potentially threatening events, may lead to depression or anxiety respectively (Muris, 2002). Selfefficacy is however separate from self-esteem (Bandura, 1997), which itself has been suggested to be critical for positive SWB through positive appraisal of the self (Diener, 1984), and optimism, which represents a tendency to expect a favourable outcome and may affect SWB through the increase in behaviours that may lead to more positive experiences through the successful attempt at, and attainment of, goals (Lucas, Diener, \& Suh, 1996). Successful identification of the relevant factors associated with well-being in a particular environment should therefore involve personality factors as well as factors related to circumstances or environment.

\subsection{Differential Effects}

It is important to note that while studies often pick one aspect of SWB and report findings as representative of SWB as a whole (Diener, Oishi, \& Lucas, 2003), satisfaction, positive affect, and negative affect have been demonstrated to be discriminant factors that should be measured individually (Busseri, Sadava, \& DeCourville, 2007; Diener et al., 2003; Lucas et al., 1996). Using multitrait-multimethod analysis, Lucas et al. (1996) demonstrated the distinction between life satisfaction and affective components of SWB, along with the existence of positive and negative affect as individual elements rather than two ends of the same spectrum. Buserri et al. (2007) confirmed that while each component of SWB has common variance, they also have significant unique variance. Not only does this suggest that these factors should be measured as separate elements of SWB, but also that the relationships between predictor variables and SWB may vary according to which particular element of SWB is measured (Busseri et al., 2007). This has important implications for how well-being is measured in practice, such that measuring only the absence of negative outcomes or certain elements of SWB may lead to conclusions regarding the overall well-being of employees that may be inaccurate, or underestimation of the impact of particular circumstances.

\subsection{Practical Measurement}

In order to achieve positive well-being or reduce negative well-being it is critical to be able to identify the contributory factors, so that any source of poor well-being can be identified and removed and any source of positive well-being can be applied elsewhere. Furthermore, it has been suggested that research on SWB must take into account interactions between the situation and the individual and the differential influences of variables on each component of SWB (Diener et al., 1999). In terms of well-being in the workplace, the measurement of predictors including work characteristics and individual differences would account for the separate influences and interactions of environment and personality. Measuring potential contributory factors in this way would provide an approach which identifies potential causes of positive or negative well-being as a basis for action. However, accounting for the range of potentially significant factors becomes impractical when established scales of these measures are used, due to the emphasis on multiple-item measures of any factor.

The academic community emphasises the use of multi-item scales in order to provide the best likelihood of satisfactory validity and reliability (Wanous \& Hudy, 2001), however while this approach focuses on providing a psychometrically robust measure, the practicality of measuring multiple factors is sacrificed. Using this approach when taking into account the range of well-being relevant factors such as those described above can lead to questionnaires that are very time consuming and costly in terms of data collection and analysis. The solution may therefore be to reduce the number of different variables measured in order to reduce the impact on time, costs, and potential for respondent refusal, however as the above research suggests, this may lead to potentially 
important factors being left out or findings related to one element of well-being inappropriately applied to well-being as a whole. One alternative is to use single-item measures, allowing for a large reduction in the number of overall questions while maintaining the number of domains measured, thus providing a practical solution for organisations to identify and target areas for improvement from a range of potential issues.

\subsection{Single-Items}

Although single-item measures are often discouraged for research based on traditional concerns regarding their psychometric properties, the issues regarding application of research to practical settings such the workplace has led to some investigation into their appropriateness when circumstances restrict the length of the measurement design (Bowling, 2005; De Boer et al., 2004; Littman, White, Satia, Bowen, \& Kristal, 2006). Research by Wanous and colleagues (Wanous \& Hudy, 2001; Wanous et al., 1997) for instance has challenged the negative assumptions often made about single item measures, showing that single item reliability is often estimated within the boundaries of satisfactory reliability. One example is in the measurement of job satisfaction where the results of a meta-analysis showed a minimum estimated reliability of .70 for single item job satisfaction measures (Wanous et al., 1997). Other studies (Scarpello \& Campbell, 1983) have suggested that a single item measure of overall job satisfaction was preferable to a scale that is based on a sum of specific job facet satisfactions and Wanous et al. (1997) conclude that the use of single items should not be discounted and should be investigated in further applications.

In terms of workplace well-being, Williams (2012) showed that single-item measures of demands-controlsupport and effort-reward imbalance models showed strong correlations with the multi-item scales, and very similar relationships with happiness, depression, anxiety, and positive and negative affect, in terms of the Pearson R correlations and the mean scores on these outcomes for high, medium, and low-risk groups based on the model predictions. This provided initial support to the idea that single-item measures can be useful for workplace well-being when time and costs are restricted.

The purpose of the current study is to further examine whether single items provide an appropriate way of applying research on well-being into practice, accounting for the simultaneous effect of multiple factors in order to determine the independent and combined effects of environment and personality, while maintaining practical considerations in terms of survey length. Based on the literature regarding the importance of situational factors and personality factors, along with the potential for interaction effects and variability in significance depending on outcome measure, the following hypotheses are tested:

- Ratings of emotional stability, optimism, self-efficacy, self-esteem provide a significant predictor of outcomes, beyond that of demands, control, support, effort, and reward.

- Relationships between predictors and cognitive and emotional outcomes will vary, in that significant predictors of one outcome will not necessarily apply to all outcomes-representing the discrimination of outcomes.

- Testing predictions from the top-down perspective, one or all personality factors will interact with one or all work factors in their relationships with outcomes.

\section{Method}

\subsection{Participants}

Twenty-eight male and 87 female university staff members aged 20 - 64 participated in the study, as part of a larger survey on well-being measurement. This number of participants was considered satisfactory to provide a meaningful cases-to-IV ratio for multiple regression analysis (Tabachnick \& Fidell, 2007). The majority of the sample were aged 30 - 39 (31.6\%), married or living with a partner (62.5\%), educated to degree or higher degree level (73.33\%), and earned between $£ 10,000$ - £19,999 per year. Participants from all areas of the university were able to participate, including finance, teaching, accommodation, and security, although the actual number of respondents from each service were not recorded.

\subsection{Materials-Single-Item Measures}

Single-item measures were developed using a format that was designed to provide a single item for each domain of a multi-item scale, while keeping ambiguity to a minimum. To meet these criteria, each single item contained an initial statement which the respondent was required to reply to on a 10 point scale from Strongly Disagree to 
Strongly Agree. Following each statement was a list of examples of what the respondent was to consider when making their response, containing examples from the corresponding multi-item scale (see self-efficacy example in Figure 1). At the beginning of the questionnaire, participants were instructed that: "Many of these questions will contain examples of what thoughts/behaviours the question is referring to, which are important for understanding the focus of the question but should be regarded as guidance rather than strict criteria." Depending on the format of the comparison multi-item scale, one or two items were used for each element. This resulted in one item for each of the following domains: Demands, Control, Support, Effort, Self-Efficacy. Two items were used for Optimism, Self-Esteem, and Emotional Stability, providing a positive and negatively worded question to represent the positive and negative aspects of the multi-item scales they were based on, and finally two items were used for Reward, one representing financial reward and one representing esteem reward. In accordance with the scoring criteria of the multi-item scales, the two-item measures were combined to provide a total score for each element.

\subsection{Multi-Item Scales}

Multi-item scale versions of the single-item measures were included in the questionnaire as a comparison for the single-item versions. These included: The Demand Control Support Questionnaire (Sanne, Torp, Mykletun, \& Dahl, 2005), the effort and reward sections of the effort-reward imbalance questionnaire (Siegrist et al., 2004), the Life Orientation Test-Revised (Optimism) (Scheier, Carver, \& Bridges, 1994), the Rosenberg Self-Esteem Scale (Rosenberg, 1965), and the General Self-Efficacy Scale (Schwarzer \& Jerusalem, 1995).

\subsection{Outcome measures}

Self-reported anxiety and depression were measured using the Hospital Anxiety and Depression Scale (Zigmond \& Snaith, 1983). The scale contains 14 items, 7 relating to symptoms of depression and 7 relating to symptoms of anxiety. Participants respond to each item on a scale from 0 to 3 and items for each subscale are summed so that higher scores represent greater negative symptoms.

Positive and negative affect were measured using the international short-form positive and negative affect schedule (I-PANAS-SF) (Thompson, 2007). Participants rate to what extent they feel positive or negative feelings in terms of 5 positive and 5 negative items such as Upset and Alert. Responses are made on a 5 item scale from Never to Always.

Job satisfaction was measured using a single item from the Well-being module of the European Social Survey (Huppert et al., 2009): “All things considered, how satisfied are you with your present job”, where participants marked their agreement on a scale from 1 "Extremely Dissatisfied” to 10 "Extremely Satisfied".

\subsection{Design}

A cross-sectional design was used, where all participants completed all sections of the questionnaire online.

\subsection{Procedure}

Participants responded to an internal advertisement on the university online notice board and those interested were sent a link to an online questionnaire which they could complete in their own time and took approximately one hour to complete. Participants were instructed that they could skip any questions that they were not comfortable answering, although all data was provided anonymously. The questionnaire itself began with demographics, followed by job characteristics; the developed short items; and the full scales. All data were collected electronically using Survey Tracker software and scored and analysed using SPSS 18 and Microsoft Excel.

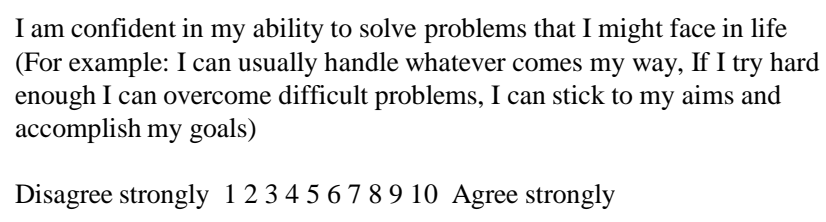

Figure 1. The single item self-efficacy question. 


\section{Results}

\subsection{Validity of Single-Item Measures}

In order to assess initial validity of the single-item measures, correlations were made between these and their respective multi-item scales. Correlations for the single-item work factors can be seen in Williams (2012). Correlations for the personality factors ranged from .57 (emotional stability) to .80 (optimism) with their respective multi-item scale and from .29 (self efficacy-emotional stability) to .70 (self esteem-optimism) for between-item comparisons of the single-item measures. For comparison, between-item correlations using the multi-item scales alone ranged from .43 (self efficacy-emotional stability) to .76 (self esteem-optimism).

\subsection{Work Characteristics}

Variables were assessed for skewness and kurtosis based on guidance in Tabachnick and Fidell (2007) and transformations were made for any variable with a skewness or kurtosis of .7 or more. This resulted in the square root transform being used for: support, optimism, self efficacy, self esteem, emotional stability (negatively skewed) and depression (positively skewed). Distributions of the transformed variables showed that the square root transform was satisfactory, bringing skewness and kurtosis to more desirable levels.

\subsection{Factor Analysis}

In order to reduce the number of variables entered into multiple regression analysis, independent predictor variables were combined into total scores based on factor analysis. Factor analysis was performed for work characteristics and personality factors separately in order to maintain logically distinct groups.

\subsection{Work Characteristics}

Effort, reward, demands, control and support were entered using initial criteria based on a minimum eigenvalue of 1 . This resulted in two factors being extracted accounting for $73.6 \%$ of the variance, however the noticeable break in the scree plot was clearly at 3 factors, so based on the guidance provided in Tabachnick and Fidell (2007) analysis was repeated using 3 factors as the criteria, providing a solution that accounted for $84.5 \%$ of the variance. Rotation using the Oblimin method with Kaiser normalization provided a 3 component pattern, comprising of Reward and Support (factor 1-positive work characteristics), Effort and Demands (factor 2-negative work characteristics), Control (factor 3-control), each with loadings greater than .85.

\subsection{Personality}

Optimism, self-efficacy, self-esteem, and emotional stability were entered using an initial criteria based on a minimum eigenvalue of 1 . This resulted in one factor extracted accounting for $60.89 \%$ of the variance. A noticeable break in the scree plot was however present at 2 factors, and so analysis was repeated using 2 factors as the criteria. This provided a solution that accounting for $80.49 \%$ of the variance providing self efficacy, optimism, self esteem (factor 1-positive self attitude) and emotional stability (factor 2-emotional stability), each with loadings greater than .80 .

The factor analysis resulted in the creation of 5 factors: Positive work characteristics (PWC), negative work characteristics (NWC), control (CONT), positive self attitude (PSA), and emotional stability (ES). Each of these factors was assessed for normality, resulting in the square root transformation being used on positive work characteristics to reduce negative skewness (-.966).

\subsection{Correlations}

In order to assess initial relationships with outcomes, the predictor variables and combined factors were correlated with outcome variables. Pearson R correlations for the relationships between predictors and outcomes are summarised in Table 1 (work circumstances), Table 2 (personality factors) and Table 3 (combined factors) below:

In summary, effort and demand show the greatest variability in the significance of their relationships with outcomes, with effort showing significant correlations with depression and anxiety but no other variables, while 
Table 1. Shows correlations between work circumstances and well-being outcomes (Pearson R).

\begin{tabular}{cccccc}
\hline & $\begin{array}{c}\text { Demands } \\
(\mathrm{n}=118)\end{array}$ & $\begin{array}{c}\text { Control } \\
(\mathrm{n}=120)\end{array}$ & $\begin{array}{c}\text { Support } \\
(\mathrm{n}=119)\end{array}$ & $\begin{array}{c}\text { Effort } \\
(\mathrm{n}=120)\end{array}$ & $\begin{array}{c}\text { Reward } \\
(\mathrm{n}=120)\end{array}$ \\
\hline Depression & .147 & $-.301^{* *}$ & $-.336^{* *}$ & $.318^{* *}$ & $-.288^{*}$ \\
Anxiety & .100 & $-.345^{* *}$ & $-.246^{* *}$ & $.206^{*}$ & -.139 \\
Positive Affect & -.094 & $.308^{* *}$ & $.205^{*}$ & -.059 & $.255^{* *}$ \\
Negative Affect & .070 & $-.286^{* *}$ & $-.279^{* *}$ & .108 & $-.239^{* *}$ \\
Job Satisfaction & $-.209^{*}$ & $.507^{* *}$ & $.490^{* *}$ & -.160 & $.631^{* *}$ \\
\hline
\end{tabular}

* Significant at $.05,{ }^{* *}$ Significant at .01.

Table 2. Shows correlations between personality factors and well-being outcomes (Pearson R).

\begin{tabular}{ccccc}
\hline & $\begin{array}{c}\text { Optimism } \\
(\mathrm{n}=120)\end{array}$ & $\begin{array}{c}\text { Self-Efficacy } \\
(\mathrm{n}=117)\end{array}$ & $\begin{array}{c}\text { Self-Esteem } \\
(\mathrm{n}=120)\end{array}$ & $\begin{array}{c}\text { Emotional Stability } \\
(\mathrm{n}=120)\end{array}$ \\
\hline Depression & $-.606^{* *}$ & $-.369^{* *}$ & $-.543^{* *}$ & $-.336^{* *}$ \\
Anxiety & $-.524^{* *}$ & $-.389^{* *}$ & $-.549^{* *}$ & $-.417^{* *}$ \\
Positive Affect & $.645^{* *}$ & $.511^{* *}$ & $.569^{* *}$ & $.313^{* *}$ \\
Negative Affect & $-.494^{* *}$ & $-.336^{* *}$ & $-.475^{* *}$ & $-.534^{* *}$ \\
Job Satisfaction & $.389^{* *}$ & $.234^{*}$ & $.218^{*}$ & $.238^{* *}$ \\
\hline
\end{tabular}

*Significant at $.05,{ }^{* *}$ Significant at .01 .

Table 3. Shows correlations between combined factors and well-being outcomes (Pearson R).

\begin{tabular}{cccccc}
\hline & $\begin{array}{c}\text { NWC } \\
(\mathrm{n}=118)\end{array}$ & $\begin{array}{c}\text { PWC } \\
(\mathrm{n}=119)\end{array}$ & $\begin{array}{c}\text { CONT } \\
(\mathrm{n}=120)\end{array}$ & $\begin{array}{c}\text { PSA } \\
(\mathrm{n}=117)\end{array}$ & $\begin{array}{c}\text { ES } \\
(\mathrm{n}=120)\end{array}$ \\
\hline Depression & $.256^{* *}$ & $-.249^{* *}$ & $-.301^{* *}$ & $-.613^{* *}$ & $-.336^{* *}$ \\
Anxiety & .165 & -.155 & $-.345^{* *}$ & $-.583^{* *}$ & $-.417^{* *}$ \\
Positive Affect & -.080 & $.258^{* *}$ & $.308^{* *}$ & $.682^{* *}$ & $.313^{* *}$ \\
Negative Affect & .098 & $-.251^{* *}$ & $-.286^{* *}$ & $-.522^{* *}$ & $-.534^{* *}$ \\
Job Satisfaction & $-.211^{*}$ & $.636^{* *}$ & $.507^{* *}$ & $.329^{* *}$ & $.238^{* *}$ \\
\hline
\end{tabular}

*Significant at $.05,{ }^{* *}$ Significant at .01 .

demands shows a significant negative correlation with job satisfaction alone. Rewards, control, and support each have more consistently significant correlations with the variety of outcomes.

In terms of personality, each predictor is significantly correlated with all outcomes, however the strength of these relationships does vary according to outcome variable, particularly in terms of a stronger correlation with emotional outcomes than with job satisfaction.

When the predictor variables are combined into factors a similar pattern of results is seen, with NWC being most significantly correlated with depression and job satisfaction, PWC also correlating significantly with positive and negative affect, while CONT, PSA, and ES were significantly correlated with all outcomes.

\subsection{Multiple Regression}

Multiple regressions were performed using the combined factors and each individual outcome measure in order to determine what the significant unique predictors of each outcome were. Only those predictor variables that had significant correlations with outcomes were included in the regression analysis, resulting in the following comparisons: NWC, PWC, CONT, PSA, ES on depression; CONT, PSA, ES on anxiety; PWC, CONT, PSA, 
ES on positive affect; PWC, CONT, PSA, ES, on negative affect; NWC, PWC, CONT, PSA, ES, on job satisfaction.

Tables 4-8 show the results for the multiple regression analyses for each outcome variable. In summary, the significant unique predictors of outcomes were: NWC (depression), PWC (job satisfaction), CONT (anxiety, positive affect (.05), job satisfaction), PSA (depression, anxiety, positive affect, negative affect, job satisfaction), and ES (anxiety, negative affect).

\subsection{Interactions}

Mediation analyses were performed to test the hypothesis that the relationship between environmental factors and outcomes is dependent on personality factors. The SOBEL (Preacher \& Hayes, 2004) approach to mediation analysis was used, which reports each of the correlations between predictor, mediator, and outcome, the significance of the partial correlation when the mediator is taken into account, and the $95 \%$ confidence intervals of the mediation after bootstrapping (in this case with 3000 samples). Mediations were performed based on those factors that had a significant correlation with outcomes, with PSA as the mediator. This resulted in analyses for PWC, NWC, and CONT with depression, CONT with anxiety, PWC, and CONT with positive affect and negative affect, and NWC, PWC, and CONT with job satisfaction. No significant mediation was found with any combination of predictor and outcome variable.

Table 4. Shows unstandardized beta coefficient, 95\% confidence intervals, and model summary for significant predictor variables on depression.

\begin{tabular}{ccc}
\hline & Depression & \\
\hline Variable & $\mathrm{B}$ & $95 \% \mathrm{CI}$ \\
\hline Constant & 3.551 & {$[2.856,4.245]$} \\
NWC & $.036^{*}$ & {$[.002, .069]$} \\
PWC & -.014 & {$[-.047, .020]$} \\
CONT & -.075 & {$[-.154, .003]$} \\
PSA & $-.280^{* *}$ & {$[-.363,-.197]$} \\
ES & -.063 & {$[-.280, .154]$} \\
R & .448 & \\
F & $17.695^{* *}$ & \\
\hline
\end{tabular}

*Significant at $.05,{ }^{* * *}$ Significant at .01 .

Table 5. Shows unstandardized beta coefficient, 95\% confidence intervals, and model summary for significant predictor variables on anxiety.

\begin{tabular}{ccc}
\hline & Anxiety & \\
\hline Variable & B & $95 \%$ CI \\
\hline Constant & 17.847 & {$[15.403,20.291]$} \\
CONT & $-.493^{* *}$ & {$[-.792,-.194]$} \\
PSA & $-1.079^{* *}$ & {$[-1.439,-.719]$} \\
ES & $-.987^{*}$ & {$[-1.925,-.049]$} \\
$\mathrm{R}^{2}$ & .427 & \\
F & $28.039^{* *}$ & \\
\hline
\end{tabular}

*Significant at $.05,{ }^{* *}$ Significant at .01 . 
Table 6. Shows unstandardized beta coefficient, 95\% confidence intervals, and model summary for significant predictor variables on positive affect.

\begin{tabular}{ccc}
\hline & Positive Affect & \\
\hline Variable & B & $95 \%$ CI \\
\hline Constant & 10.823 & {$[9.132,12.515]$} \\
PWC & .064 & {$[-.035, .162]$} \\
CONT & $.231^{*}$ & {$[.000, .462]$} \\
PSA & $1.083^{* *}$ & {$[.841,1.324]$} \\
ES & -.105 & {$[-.742, .532]$} \\
R & .512 & \\
F & $29.332^{* *}$ & \\
\hline
\end{tabular}

*Significant at $.05,{ }^{* *}$ Significant at .01 .

Table 7. Shows unstandardized beta coefficient, 95\% confidence intervals, and model summary for significant predictor variables on negative affect.

\begin{tabular}{ccc}
\hline \multicolumn{3}{c}{ Negative Affect } \\
\hline Variable & B & $95 \%$ CI \\
\hline Constant & 17.410 & {$[15.611,19.208]$} \\
PWC & -.034 & {$[-.139, .071]$} \\
CONT & -.202 & {$[-.448, .044]$} \\
PSA & $-.549^{* *}$ & {$[-.806,-.292]$} \\
ES & $-1.445^{* *}$ & {$[-2.112,-.768]$} \\
R & .416 & \\
F & $19.973^{* *}$ & \\
\hline
\end{tabular}

*Significant at $.05,{ }^{* *}$ Significant at .01 .

Table 8. Shows unstandardized beta coefficient, 95\% confidence intervals, and model summary for significant predictor variables on job satisfaction.

\begin{tabular}{ccc}
\hline & Job Satisfaction & \\
\hline Variable & $\mathrm{B}$ & $95 \% \mathrm{CI}$ \\
\hline Constant & .781 & {$[-.773,2.335]$} \\
NWC & -.055 & {$[-.130, .020]$} \\
PWC & $.233^{* *}$ & {$[.157, .308]$} \\
CONT & $.239^{* *}$ & {$[.062, .415]$} \\
PSA & $.280^{* *}$ & {$[.094, .466]$} \\
ES & -.065 & {$[-.550, .421]$} \\
R & .509 & \\
F & $22.565^{* *}$ & \\
\hline
\end{tabular}

*Significant at $.05,{ }^{* *}$ Significant at .01 . 
Secondly, moderation analyses were performed in order to identify any relationships between environmental factors and outcomes that may be moderated by personality factors. Based on guidance in Aiken \& West (1991), centred variables along with the interaction variables were entered on multiple regression for NWC, PWC, CONT and PSA on each outcome measure. A significant relationship was found for the CONT PSA interaction on positive affect, and this interaction is plotted in Figure 2, using \pm 1 standard deviation as the low and high values of CONT and PSA, and in Figure 3 using the minimum and maximum values for a clearer picture. As can be seen, PSA moderates the relationship between CONT and positive affect, such that at low and medium levels of PSA, those lowest in CONT have the lowest positive affect, however at the highest level of PSA, the difference between levels of CONT and positive affect is reduced.

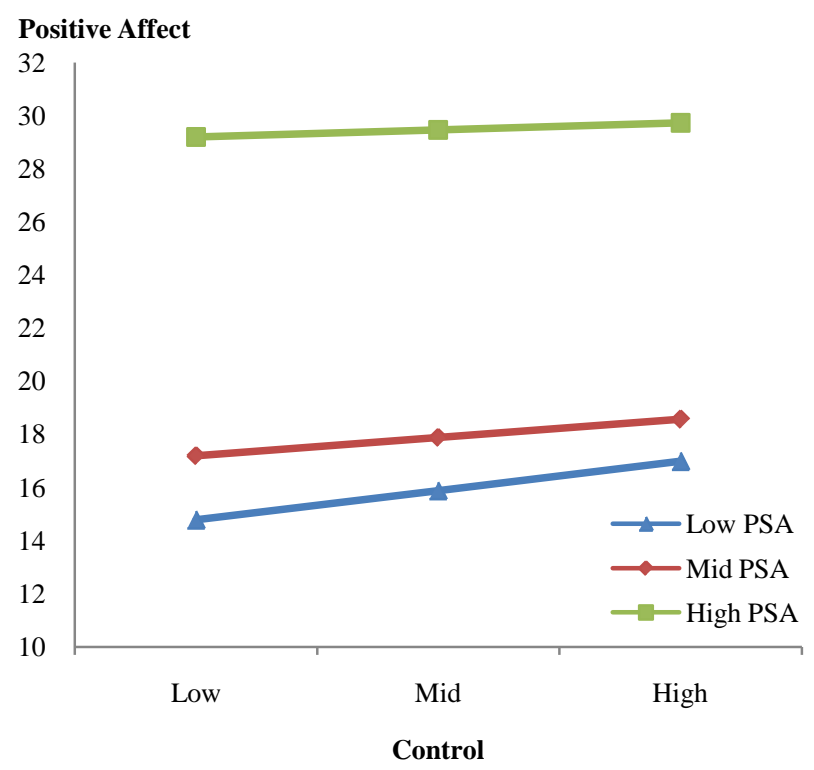

Figure 2. Interaction between control and positive self-attitude: Positive Affect (based on \pm 1 standard deviation as the low and high values of CONT and PSA).

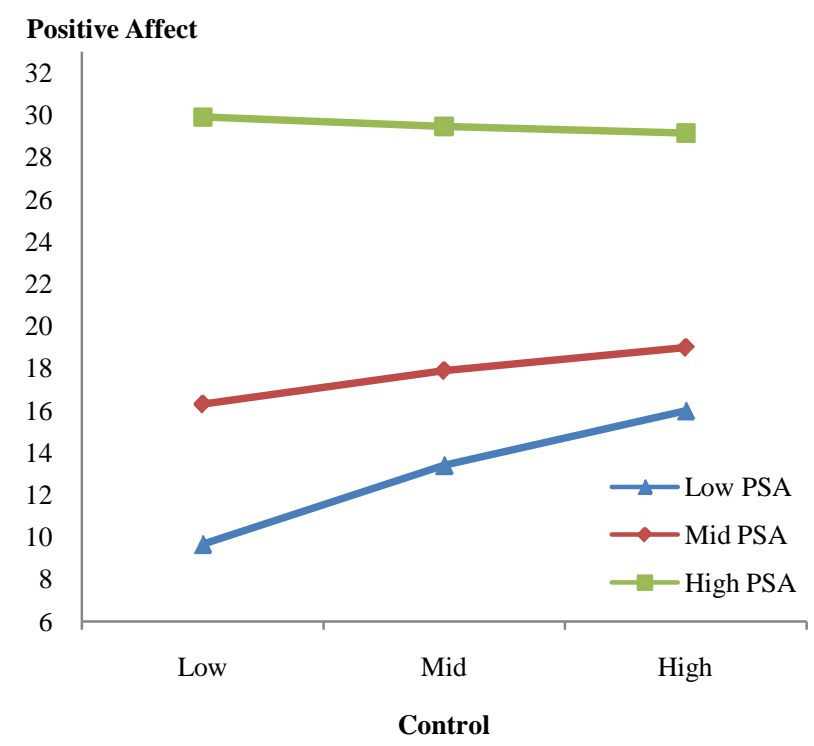

Figure 3. Interaction between control and positive self-attitude: Positive Affect (using the minimum and maximum as the low and high values of CONT and PSA). 
Due to the consistent significance of PSA over ES as a predictor of outcomes, mediation analyses were also performed for PSA as a mediator of the relationship between ES and outcomes. The results showed that PSA fully mediated the effect of ES on depression and positive affect, and partially mediated the effect of ES on anxiety and negative effect. No mediation was found for ES and job satisfaction.

\section{Discussion}

The results indicate that environmental work factors and personality factors have varying relationships with well-being outcomes. Correlations show that work characteristics seem to have a greater effect on cognitive judgement of job satisfaction than emotional well-being factors, however the accuracy of this conclusion varies according to which work-specific factor is investigated, with control in particular being significantly related to all aspects of well-being. Negative work characteristics such as demands and effort, however, seem to only have comparatively weaker relationships with depression and job satisfaction.

When multiple-regression analysis is employed, results suggest that personality factors explain a consistently greater amount of variance in outcome measures, suggesting that personality is more useful than environmental factors in predicting well-being, supporting numerous proponents of this possibility. However the significance of personality also varies depending on whether positive self appraisal or emotional stability is used, with emotional stability predicting a significant amount of unique variance in anxiety and negative affect, but not depression, positive affect or job satisfaction, while PSA was a significant predictor of all outcome measures. Although ES also correlated significantly with depression and positive affect, this relationship was mediated by PSA, suggesting that emotional stability affects these outcomes through its effect on optimism, self-esteem, and self-efficacy. This is also partially true for the relationship between emotional stability, anxiety and negative affect, although ES still maintains a significant unique contribution to these outcomes.

The results provide support for each of the main hypotheses and the use of single-items to measure well-being in practice. Firstly, the results suggest that emotional and cognitive well-being outcomes should indeed be measured independently, because the same predictor variables can have different relationships with well-being depending on the measure used, as suggested by Brief et al. (1993). This was noticeable throughout the analyses, with variations in the significance of correlations depending on outcome, and similar variations in significant predictors using multiple regression analyses. In particular, it was most noticeable, although not unexpected based on the greater specificity of job satisfaction as an outcome, that work characteristics in general were important factors for cognitive appraisal of job satisfaction, but less so for emotional well-being outcomes. This difference in relationships also varies within work characteristics however, with negative work characteristics predicting a significant amount of unique variance in depression, and control also having a significant relationship with anxiety and positive affect. The results also provide support for emotional stability being a predictor of negative emotional well-being over positive emotional well-being (Costa \& McCrae, 1980). The significant mediation effect found for PSA and ES partially supports findings by Strobel, Tumasjan, and Spörrle (2011), who found that self-efficacy mediated the relationship between extraversion and neuroticism on SWB. However, in their study, the effect was significant for life satisfaction but non-significant for happiness, leading to the conclusion that the mediation relationship was more relevant for the cognitive aspect of SWB. The contrasting results found in the current study are likely due to the difference in the constructs measured, in terms of the inclusion of optimism and self-esteem in the mediator variable, and the use of job satisfaction as an outcome rather than life satisfaction, along with the use of other emotional well-being outcomes instead of happiness. The results may therefore have been different if the same measures were used, however Cheng and Furnham (2003) also found that self-esteem mediated the effect of neuroticism and extraversion on happiness and depression among adolescents. Overall, the results support previous suggestions that a relationship in one aspect of well-being does not necessarily apply to other aspects (Busseri et al., 2007).

Secondly, ratings of personality in terms of the combined effect of optimism, self-efficacy, and self-esteem provided significantly greater predictor of all outcomes than the combined effects of work characteristics in demands, control, support, effort, and reward, as PSA predicted a significant amount of variance over and above other factors for each outcome measure. Furthermore, emotional stability also had significant relationships with outcome measures in terms of correlations, however only predicted unique variance of two of the five outcome measures, suggesting that while personality factors in general may offer significant prediction of well-being outcomes, the particular aspect of personality that is measured also matters, with the combination of optimism, 
self-efficacy, and self-esteem providing the most consistent effects. The fact that PSA and ES explained unique variance in some outcomes is particularly interesting as it provides support for evidence that optimism, self-esteem, and self-efficacy, at least as a combined group, may not necessarily just be manifestations of neuroticism as has been previously suggested may be the case (Diener et al., 2003; Lucas et al., 1996). It also has implications for application in practice, such that the influence of personality may vary depending on the personality measure used. Despite the apparent significance of personality factors, the results also show that work related environmental factors also contribute to the prediction of outcomes and should not be discounted, particularly in respect to positive work characteristics such as support and reward, along with control. This supports previous research which suggests that a combination of various factors provides the best predictor of positive and negative outcomes (Smith \& Wadsworth, 2011; Williams \& Smith, 2012), and demonstrates the ability for these single-items to discriminate the effects of varying predictors.

Thirdly, the top-down assumption of an interaction between personality and environmental factors was partially supported, although it appears that this also depends on the particular variables measured. A significant moderation interaction was found for the relationship between control and positive self attitude, but only when positive affect was the outcome. The interaction showed that personality determined whether or not environmental factors influenced well-being outcomes, such that the level of PSA influenced the effect that control had on positive affect. More specifically, those with high control are better off in terms of positive affect than those with low control, however this is only the case when optimism, self-efficacy, and self-esteem are at low or moderate levels. At the higher end of PSA, the effect of control on positive affect is diminished, and at the highest levels those with the highest control have slightly lower positive affect. This may suggest that at significantly high enough PSA the positive effect of control is no longer important, or potentially that high PSA buffers the negative effect of low control on positive mood, and demonstrates the importance of taking personality into account when testing assumptions based on the role of work factors in well-being. The result also has implications in terms of practical application, such that while those with low or mid levels of these personality factors may benefit from high control, those high in these personality factors may not benefit from an intervention designed to increase control.

Finally, the results in this study supporting multiple hypotheses based on the literature, and demonstrating discrimination between variables and complex relationships have been found using single or two-item scales for the predictor variables, suggesting that this approach to practical measurement of well-being does provide an economical way of gaining valuable information related to well-being in situations where time, costs, or response burden are of particular importance. Initial validation showed significant correlations between singleitem and multi-item measures of the same constructs, and although significant correlations were found between separate factors, the strength of these correlations was actually lower than those found using multi-item measures, suggesting that the single-items discriminate between similar factors at least as well as, if not better than, the multi-item scales. The one possible exception to this is emotional stability, where the single item showed the smallest correlation with its multi-item counterpart (.57) compared to the other factors, and between-item correlations ranged from .36 to .51 . This may suggest that the lack of unique variance demonstrated by ES in the multiple-regression analyses may be an artefact of the single-item measure, and that a more discriminant emotional stability measure may provide more independent relationships.

\section{Limitations}

Although the findings presented here suggest that personality factors, particularly optimism, self-efficacy, and self-esteem, are important predictors of well-being in the workplace, and that single-item measures in the format used provide a practical way of measuring these factors, it is important that they are taken in context. Firstly, the role of particular personality factors may depend on culture, particularly in terms of the relationship between satisfaction with the self (i.e. self esteem) and the level of individualism across cultures (Diener et al., 2003), meaning that the effect found for PSA in the current study may not be as profound in more individualistic populations. Another effect that the sample may have had on the results is related to the format of the questions. The single-item measures used required participants to consider examples taken from the multiple-item scales and make overall judgements of how much they agreed or disagreed with the item. Over $85 \%$ of participants were educated to AS-level or above with over $70 \%$ to degree level, and it's possible that those with a less academic background may not have the knowledge of terms used to be able to make judgements as accurately. This is par- 
ticularly important for single-item measures as the amount of variance due to the wording of the items is not averaged out (Diener, 1984), although a number of examples are given in the single-items to allow for a general idea if one example is not understood.

In terms of methodology, the methods used (i.e. correlation and multiple regression), while providing evidence of the strength of the relationships between variables, do not provide more in-depth information regarding the specific nature of those relationships. In this study, this particularly refers to the causal nature of personality factors and outcomes, for example low job satisfaction or low mood may lead to lower ratings of self-esteem or optimism. Furthermore, high inter-item correlations (particularly between self-esteem and optimism) suggests that perhaps further examination of particular items may reveal redundant measures, as the similar multi-item correlation between these two factors suggests it is not an artefact of single-item measurement.

\section{Conclusion}

In conclusion, the results indicate that the measurement of well-being and its constituent elements is complex, with a variety of potential contributors to negative or positive well-being depending on the outcome measured. While work related factors appear to have particular importance when positive aspects of the work and level of control is considered, measurement of well-being predictors in the workplace should also take into account personality factors and take care not to assume relationships in one aspect of well-being outcomes applies to other aspects. While this conclusion may highlight the complexity of well-being measurement in practice, this complexity need not be a burden on employers with limited resources, with single-item measures providing actionable results.

\section{References}

Aiken, L. S., \& West, S. G. (1991). Multiple Regression: Testing and Interpreting Interactions. London: Sage Publications Ltd.

Bandura, A. (1997). Self Efficacy: The Exercise of Control. New York: Freeman.

Bowling, A. (2005). Just One Question: If One Question Works, Why Ask Several? Journal of Epidemiology and Community Health, 59, 342-345. http://dx.doi.org/10.1136/jech.2004.021204

Brief, A. P., Butcher, A. H., George, J. M., \& Link, K. E. (1993). Integrating Bottom-Up and Top-Down Theories of Subjective Well-Being: The Case of Health. Journal of Personality and Social Psychology, 64, 646-653. http://dx.doi.org/10.1037/0022-3514.64.4.646

Busseri, M. A., Sadava, S. W., \& DeCourville, N. (2007). A Hybrid Model for Research on Subjective Well-Being: Examining Common- and Component-Specific Sources of Variance in Life Satisfaction, Positive Affect, and Negative Affect. Social Indicators Research, 83, 413-445. http://dx.doi.org/10.1007/s11205-006-9028-8

Cheng, H., \& Furnham, A. (2003). Personality, Self-Esteem, and Demographic Predictions of Happiness and Depression. Personality and Individual Differences, 34, 921-942. http://dx.doi.org/10.1016/S0191-8869(02)00078-8

Costa, P. T., \& McCrae, R. R. (1980). Influence of Extraversion and Neuroticism on Subjective Well-Being: Happy and Unhappy People. Journal of Personality and Social Psychology, 38, 668-678. http://dx.doi.org/10.1037/0022-3514.38.4.668

De Boer, A. G. E. M., Van Lanschot, J. J. B., Stalmeier, P. F. M., Van Sandick, J. W., Hulscher, J. B. F., De Haes, J. C. J. M., et al. (2004). Is a Single-Item Visual Analogue Scale as Valid, Reliable and Responsive as Multi-Item Scales in Measuring Quality of Life? Quality of Life Research, 13, 311-320. http://dx.doi.org/10.1023/B:QURE.0000018499.64574.1f

DeNeve, K. M., \& Cooper, H. (1998). The Happy Personality: A Meta-Analysis of 137 Personality Traits and Subjective Well-Being. Psychological Bulletin, 124, 197-229. http://dx.doi.org/10.1037/0033-2909.124.2.197

Diener, E. (1984). Subjective Well-Being. Psychological Bulletin, 95, 542-575. http://dx.doi.org/10.1037/0033-2909.95.3.542

Diener, E., Oishi, S., \& Lucas, R. E. (2003) Personality, Culture, and Subjective Well-Being: Emotional and Cognitive Evaluations of Life. Annual Review of Psychology, 54, 403-425.

Diener, E., Suh, E. M., Lucas, R. E., \& Smith, H. L. (1999). Subjective Well-Being: 3 Decades of Progress. Psychological Bulletin, 125, 276-302. http://dx.doi.org/10.1037/0033-2909.125.2.276

Gargiulo, R. A., \& Stokes, M. A. (2009). Subjective Well-Being as an Indicator for Clinical Depression. Social Indicators Research, 92, 517-527. http://dx.doi.org/10.1007/s11205-008-9301-0

Häusser, J. A., Mojzisch, A., Niesel, M., \& Schulz-Hardt, S. (2010). Ten Years on: A Review of Recent Research on the Job Demand-Control (-Support) Model and Psychological Well-Being. Work and Stress, 24, 1-35. 
http://dx.doi.org/10.1080/02678371003683747

Headey, B., \& Wearing, A. (1989). Personality, Life Events, and Subjective Well-Being: Toward a Dynamic Equilibrium Model. Journal of Personality and Social Psychology, 57, 731-739. http://dx.doi.org/10.1037/0022-3514.57.4.731

Huppert, F. A., Marks, N., Clark, A., Siegrist, J., Stutzer, A., Vitterso, J., \& Wahrendorf, M. (2009). Measuring Well-Being across Europe: Description of the ESS Well-Being Module and Preliminary Findings. Social Indicators Research, 91, 301315. http://dx.doi.org/10.1007/s11205-008-9346-0

Johnson, J. V., \& Hall, E. M. (1988). Job Strain, Work Place Social Support, and Cardiovascular Disease: A Cross-Sectional Study of Random Sample of the Swedish Working Population. American Journal of Public Health, 78, 1336-1342.

http://dx.doi.org/10.2105/AJPH.78.10.1336

Karasek Jr., R. A. (1979). Job Demands, Job Decision Latitude, and Mental Strain: Implications for Job Redesign. Administrative Science Quarterly, 24, 285-308. http://dx.doi.org/10.2307/2392498

Littman, A. J., White, E., Satia, J. A., Bowen, D. J., \& Kristal, A. R. (2006). Reliability and Validity of 2 Single-Item Measures of Psychosocial Stress. Epidemiology, 17, 398-403. http://dx.doi.org/10.1097/01.ede.0000219721.89552.51

Lucas, R. E., Diener, E., \& Suh, E. (1996). Discriminant Validity of Well-Being Measures. Journal of Personality and Social Psychology, 71, 616-628. http://dx.doi.org/10.1037/0022-3514.71.3.616

Muris, P. (2002). Relationships between Self-Efficacy and Symptoms of Anxiety Disorders and Depression in a Normal Adolescent Sample. Personality and Individual Differences, 32, 337-348. http://dx.doi.org/10.1016/S0191-8869(01)00027-7

Preacher, K. J., \& Hayes, A. F. (2004). SPSS and SAS Procedures for Estimating Indirect Effects in Simple Mediation Models. Behavior Research Methods, Instruments, \& Computers, 36, 717-731. http://dx.doi.org/10.3758/BF03206553

Rosenberg, M. (1965). Society and the Adolescent Self-Image. Princeton, NJ: Princeton University Press.

Rydstedt, L. W., Devereux, J., \& Sverke, M. (2007). Comparing and Combining the Demand-Control-Support Model and the Effort Reward Imbalance Model to Predict Long-Term Mental Strain. European Journal of Work and Organizational Psychology, 16, 261-278. http://dx.doi.org/10.1080/13594320601182311

Sanne, B., Torp, S., Mykletun, A., \& Dahl, A. A. (2005). The Swedish Demand-Control-Support Questionnaire (DCSQ): Factor Structure, Item Analyses, and Internal Consistency in a Large Population. Scandinavian Journal of Public Health, 33, 166-174. http://dx.doi.org/10.1080/14034940410019217

Scarpello, V., \& Campbell, J. P. (1983). Job Satisfaction: Are All the Parts There? Personnel Psychology, 36, 577-600. http://onlinelibrary.wiley.com/doi/10.1111/j.1744-6570.1983.tb02236.x/abstract

Scheier, M. F., Carver, C. S., \& Bridges, M. W. (1994). Distinguishing Optimism from Neuroticism (and Trait Anxiety, Self-Mastery, and Self-Esteem): A Reevaluation of the Life Orientation Test. Journal of Personality and Social Psychology, 67, 1063-1078. http://dx.doi.org/10.1037/0022-3514.67.6.1063

Schwarzer, R., \& Jerusalem, M. (1995). Generalized Self-Efficacy Scale. In J. Weinman, S. Wright, \& M. Johnston (Eds.), Measures in Health Psychology: A User's Portfolio. Causal and Control Beliefs (pp. 35-37). Windsor: NFER-NELSON.

SCMH (2007). Policy Paper 8: Mental Health at Work: Developing the Business Case. London: The Sainsbury Centre for Mental Health.

Siegrist, J. (1996). Adverse Health Effects of High-Effort/Low-Reward Conditions. Journal of Occupational Health Psychology, 1, 27-41. http://dx.doi.org/10.1037/1076-8998.1.1.27

Siegrist, J., Starke, D., Chandola, T., Godin, I., Marmot, M., Niedhammer, I., \& Peter, R. (2004). The Measurement of Effort-Reward Imbalance at Work: European Comparisons. Social Science \& Medicine, 58, 1483-1499. http://dx.doi.org/10.1016/S0277-9536(03)00351-4

Smith, A. P., \& Wadsworth, E. A. (2011). A Holistic Approach to Stress and Wellbeing. Part 5: What Is a Good Job? Occupational Health (At Work), 8, 25-27.

Strobel, M., Tumasjan, A., \& Spörrle, M. (2011). Be Yourself, Believe in Yourself, and Be Happy: Self-Efficacy as a Mediator between Personality Factors and Subjective Well-Being. Scandinavian Journal of Psychology, 52, 43-48. http://dx.doi.org/10.1111/j.1467-9450.2010.00826.x

Tabachnick, B. G., \& Fidell, L. S. (2007). Using Multivariate Statistics (5th ed.). Boston, MA: Pearson Education, Inc.

Thompson, E. R. (2007). Development and Validation of an Internationally Reliable Short-Form of the Positive and Negative Affect Schedule (PANAS). Journal of Cross-Cultural Psychology, 38, 227-242. http://dx.doi.org/10.1177/0022022106297301

Tsutsumi, A., \& Kawakami, N. (2004). A Review of Empirical Studies on the Model of Effort-Reward Imbalance at Work: Reducing Occupational Stress by Implementing a New Theory. Social Science \& Medicine, 59, 2335-2359.

http://dx.doi.org/10.1016/j.socscimed.2004.03.030 
Van Der Doef, M., \& Maes, S. (1999). The Job Demand-Control (-Support) Model and Psychological Well-Being: A Review of 20 Years of Empirical Research. Work \& Stress, 13, 87-114. http://dx.doi.org/10.1080/026783799296084

Wanous, J. P., \& Hudy, M. J. (2001). Single-Item Reliability: A Replication and Extension. Organizational Research Methods, 4, 361-375. http://dx.doi.org/10.1177/109442810144003

Wanous, J. P., Reichers, A. E., \& Hudy, M. J. (1997). Overall Job Satisfaction: How Good Are Single-Item Measures? Journal of Applied Psychology, 82, 247-251. http://dx.doi.org/10.1037/0021-9010.82.2.247

Williams, G. M. (2012). Developing Short, Practical Measures of Well-Being. In M. Anderson (Ed.), Contemporary Ergonomics and Human Factors 2012 (pp. 203-210). London: Taylor \& Francis. http://dx.doi.org/10.1201/b11933-52

Williams, G. M., \& Smith, A. P. (2012). A Holistic Approach to Stress and Well-Being. Part 6: The Wellbeing Process Questionnaire (WPQ Short Form). Occupational Health (At Work), 9, 29-31.

Zigmond, A. S., \& Snaith, R. P. (1983). The Hospital Anxiety and Depression Scale. Acta Psychiatrica Scandinavica, 67, 361-370. http://dx.doi.org/10.1111/j.1600-0447.1983.tb09716.x 